DOI: http://dx.doi.org/10.35381/s.v.v3i6.305

\title{
Análisis preliminar y depuración de un circuito de acondicionamiento para un sensor electromiográfico superficial de electrodos secos diseñado in-house
}

Signal conditioning circuit debugging and preliminary analysis of an in-house designed superficial electromiography sensor

José Luis Albites Sanabria

jose.albsab@gmail.com

Universidad Nacional de Ingeniería

Universidad SISE, Lima.

Perú

https://orcid.org/0000-0001-7688-6221

Fanny Miriam Sanabria Boudri

fanny miriam@hotmail.com

Universidad Nacional Enrique Guzman y Valle

Perú

https://orcid.org/0000-0002-2462-2715

Félix José Colina Ysea

fcolina@universidadsise.edu.pe

Universidad SISE, Lima.

Perú

https://orcid.org/0000-0002-6651-3509

Recibido: 23 de enero 2019

Aprobado: 1 de marzo del 2019

\section{RESUMEN}

Diversos estudios han determinado la importancia de la electromiografía (EMG) en la detección de desórdenes musculares y de movimiento en extremidades superiores. En la actualidad, la electromiografía superficial (sEMG) se utiliza para la rehabilitación y detección de distonía, fatiga muscular, temblores entre otros fenómenos musculares. Sin embargo, la adquisición de estas señales es realizada a través de dispositivos costosos y no portátiles que requieren que el usuario asista a un laboratorio o clínica para la evaluación de estos desórdenes. Debido a ellos, empresas y laboratorios de biomédica diseñan sus propios sensores EMG in-house 
(también referido a la fabricación realizada en el mismo ambiente de trabajo). El presente estudio tiene como finalidad el análisis preliminar de los componentes de acondicionamiento analógico necesarios para el correcto funcionamiento del sensor electromiográfico a través de pruebas experimentales y de depuración utilizando instrumentos de medición electrónicos y digitales.

Descriptores: Electromiografía superficial; Condicionamiento de señales analógicas; sensores in-house; procesamiento de señales.

\section{ABSTRACT}

Several studios have determined the importance of electromiography (EMG) in the detection of movement and muscle related disorders. To the present, superficial electromyography (sEMG) is used as a rehabilitation and detection tool in muscle-related problems such as dystonia, muscle fatigue, tremors, among many others. However, currently most of the traditional and clinical equipments used in clinics are non portable and expensive. As a consequence, some companies and laboratories develop in-house electromyography sensors. The main purpose of this study is to present preliminary analysis of components and stages involved in the analog conditioning circuit of a in-house developed sensor through experimental and debugging tests using electronic and digital measurement devices.

Descriptors: Superficial electromyography, Signal conditioning, In-house sensors, Signal processing.

\section{INTRODUCCIÓN}

La ingeniería Biomédica es una rama que aplica conceptos de ingeniería a la biología y medicina para el cuidado de la salud, tanto en forma diagnóstica como terapéutica. Este campo de la ingeniería es relativamente nuevo comparado con otras ramas de ingeniería y aún gran parte de su área de trabajo consiste en desarrollo e investigación en diversos subcampos entre los que destacan: biomecánica, bioinformática, ingeniería de tejidos, neuro-ingeniería, entre otros. Asimismo, 
diversas prometedoras aplicaciones se vienen desarrollando en el campo de la ingeniería biomédica, tales como el desarrollo de dispositivos médicos avanzados que van desde micro implan- tes y nuevas drogas farmacológicas, hasta el desarrollo de prótesis biocompatibles; herramientas que provean facilidades para un mejor diagnóstico y tratamiento de diversas enfermedades (Brown, 2017).

Dentro de este campo, una diversa cantidad de equipos biomédicos se encargan de la lectura, monitoreo y diagnóstico de señales generadas por nuestro cuerpo humano. Estas señales, conocidas como señales biomédicas o bioseñales, permiten obtener información sobre el estado de diversos sistemas del cuerpo humano. Entre las bioseñales utilizadas con mayor frecuencia destacan las señales cardíacas, musculares, y en esta última década, las señales cerebrales, con estudios que muestran evidencia de personas cuadripléjicas controlando dispositivos con la mente (Nuyujukian, 2018).

Particularmente la lectura de señales musculares o electromiográficas superficiales (sEMG) es muy utilizada en condiciones que involucren el diagnóstico y tratamiento de diversas discapacidades motoras. Los sensores musculares miden la actividad eléctrica generada al contraer o relajar uno o más grupos musculares (Farina, 2016). Para la correcta medición de dicha actividad eléctrica, cada sensor muscular contiene elementos conductores, también llamados electrodos, los cuales captan estas señales. Cabe recalcar, que la actividad muscular puede capturarse tanto superficialmente (electrodos por encima de la piel) como en forma invasiva, con electrodos implantados en los nervios y fibras musculares. En el caso específico de este estudio, los sensores a utilizar son de índole superficial para minimizar cualquier intervención e interacción quirúrgica con el usuario. 


\section{Tipos y configuraciones de Electrodos}

Según Catarino (2017), los electrodos utilizados en la captura de señales superficiales se dividen principalmente en:

Electrodos con gel: son electrodos que contienen una solución líquida de plata clorada $\mathrm{Ag}-\mathrm{Cl}$. Este tipo de electrodo es muy usado en mediciones clínicas y científicas debido a su alta conductividad; baja impedancia entre piel y electrodo. Entre las desventajas se pueden mencionar, el uso de este tipo de electrodos puede resultar incómodo para el usuario. La aplicación del gel puede consumir tiempo cada vez que se desee utilizar este tipo de electrodos. Asimismo, la calidad de la señal disminuye a medida que el gel se deshidrata.

Electrodos secos: electrodos que no contienen una solución líquida. Por lo general, el material a elegir está compuesto de un metal o aleaciones de metal, entre los que destacan: oro, plata, acero inoxidable, aluminio anodizado.

Las señales musculares pueden ser registradas en forma monopolar o bipolar. La configuración monopolar hace uso de un electrodo dispuesto sobre el músculo a medir y un electrodo de referencia ubicado de preferencia lejos del músculo y en una zona inerte.

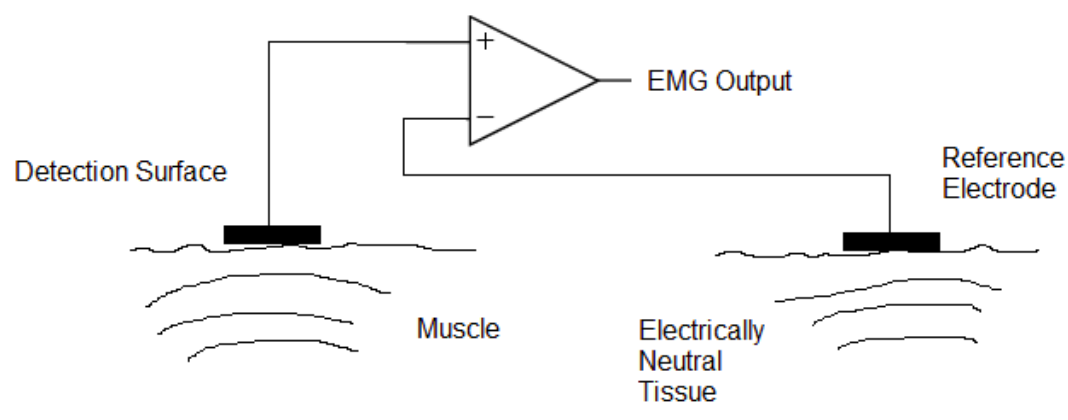

Figura 1. Configuración monopolar

La configuración bipolar consta de dos electrodos y una referencia. Por lo general, los electrodos son dispuestos sobre el músculo de interés a una distancia de entre 1-2 
cm cada uno para evitar capturar la interferencia de otros grupos musculares. La ventaja de esta configuración es la posibilidad de eliminar el ruido común entre ambos electrodos y obtener una señal muscular con menos ruido (Beck, 2017).

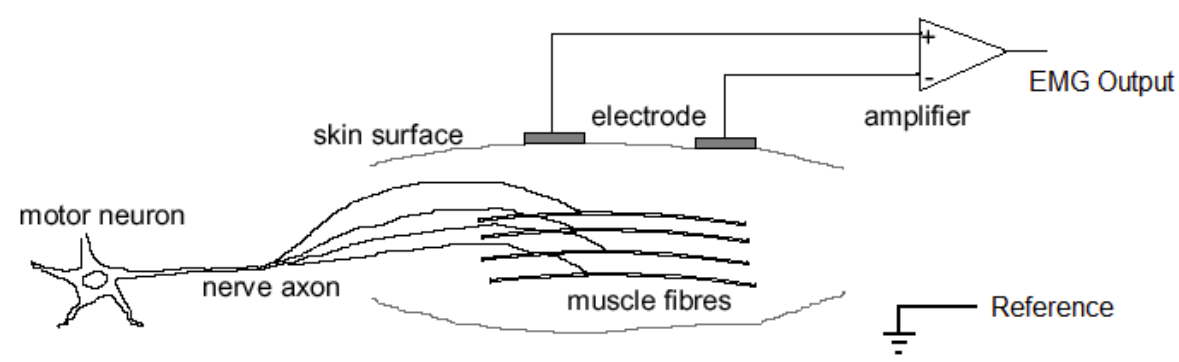

Figura 2. Configuración bipolar

Las señales musculares superficiales producen voltajes que varían entre $0-10 \mathrm{mV}$ y contienen un rango de información útil de entre $20-500 \mathrm{~Hz}$. Es recomendable que las señales obtenidas por los electrodos (sobretodo electrodos secos) sean tratadas lo más cerca posible a la zona de adquisición de la señal muscular. Esto es debido a una señal débil que viaja grandes distancias es susceptible a la inherencia de ruido a través del cable en el que viajan. Los electrodos que contienen un circuito de preprocesamiento de la señal capturada encapsulada en conjunto con el electrodo son conocidos como electrodos activos.

\section{Circuito de amplificación y preprocesamiento de señales musculares}

La configuración elegida para nuestros sensores musculares es: electrodos secos en configuración bipolar por las ventajas indicadas en la sección anterior. Según Merleti (2016), el circuito de preprocesamiento está dividido en 4 etapas de procesamiento: Pre-amplificación instrumental, Filtro pasa alto, Filtro pasa bajo, y Amplificación invertida. Esta será la configuración a utilizar y depurar en el circuito de acondicionamiento de la señal EMG. 
Revista Arbitrada Interdisciplinaria de Ciencias de la Salud. SALUD Y VIDA

Volumen 3. Número 6. Año 3. Julio - Diciembre 2019

Hecho el depósito de Ley: FA2016000010

ISSN: $2610-8038$

FUNDACIÓN KOINONIA (F.K)

Santa Ana de Coro, Venezuela.

José Luis Albites Sanabria; Fanny Miriam Sanabria Boudri; Félix José Colina Ysea

\section{METODOLOGÍA}

Se plantea un estudio experimental de investigación aplicada con un enfoque cuantitativo. La variable a ser medida es la señal que proviene de los filtros de acondicionamiento de la señal. Para ello se utilizarán instrumentos de medición de voltaje a través de un osciloscopio de marca Tektronix Tbs1032b y un generador de onda para variar la entrada al circuito de acondicionamiento de la señal a través de un generador de funciones marca BK Precision 4040B.

Con el dispositivo de funciones se generó una señal con las siguientes características: señal de tipo senoidal con un voltaje de entrada pico a pico de 8.8 mV. Las frecuencias a ser evaluadas: $10 \mathrm{~Hz}, 60 \mathrm{~Hz}, 150 \mathrm{~Hz}, 250 \mathrm{~Hz}, 350 \mathrm{~Hz}, 450 \mathrm{~Hz}$, $600 \mathrm{~Hz}, 800 \mathrm{~Hz}, 1000 \mathrm{~Hz}$.

El circuito de acondicionamiento de la señal se separó en cinco etapas: Etapa 1 referida a la amplificación diferencial (instrumental), etapa 2: filtro pasa, etapa 3: filtro pasa baja (1era etapa), etapa 4: Amplificación inversa, etapa 5: Filtro pasa baja (2da etapa). Cada prueba se repitió tres veces para asegurar la validez de la medición a través del osciloscopio.

\section{RESULTADOS}

En primera instancia, se mide la salida del amplificador instrumental y su variabilidad con diferentes fuentes de alimentación, se realizan varios tests controlando el offset a la entrada del circuito. 


\section{Test 1}

Se realiza la primera prueba utilizando una señal con las características escogidas en forma aleatoria de $356 \mathrm{~Hz}, 6.5 \mathrm{mV}$, Senoidal, offset de entrada: $0+-1 \mathrm{mV}$. El offset se varía en intervalos de $5 \mathrm{mV}$.

Tabla 1. Medición de la Salida test 1 por cada etapa

\begin{tabular}{|c|c|c|c|c|c|c|}
\hline $\begin{array}{c}\text { Voltaje de } \\
\text { alimentación }\end{array}$ & & $\begin{array}{c}\text { 1ra } \\
\text { etapa }\end{array}$ & $\begin{array}{c}2 d a \\
\text { etapa }\end{array}$ & $\begin{array}{c}\text { 3ra } \\
\text { etapa }\end{array}$ & $\begin{array}{c}4 \text { ta } \\
\text { etapa }\end{array}$ & $\begin{array}{c}\text { Después del } \\
\text { offset }\end{array}$ \\
\hline$+-3.3 V$ & $\begin{array}{l}\text { Vpp } \\
\text { Offset }\end{array}$ & $\begin{array}{l}110 \mathrm{mv} \\
10 \mathrm{mV}\end{array}$ & $\begin{array}{c}142 \mathrm{mV} \\
15+-5 \mathrm{mV}\end{array}$ & $\begin{array}{l}4.16 \mathrm{~V} \\
-648 \mathrm{mV}\end{array}$ & $\begin{array}{c}4.88 \\
-820 \mathrm{mV}\end{array}$ & $\begin{array}{c}1.68 \mathrm{Vpp} \\
908 \mathrm{~m}\end{array}$ \\
\hline$+-5.0 \mathrm{~V}$ & $\begin{array}{l}\text { Vpp } \\
\text { Offset }\end{array}$ & $\begin{array}{l}110 \mathrm{mV} \\
8+-4 \mathrm{mV}\end{array}$ & $\begin{array}{c}140 \mathrm{mV} \\
16 \mathrm{mV}+-3\end{array}$ & $\begin{array}{c}4.12 \\
-649 m V\end{array}$ & $\begin{array}{c}4.88 \\
-802 \mathrm{mV}\end{array}$ & $\begin{array}{c}1.76 \mathrm{~V} \\
935 \mathrm{mV}\end{array}$ \\
\hline$+-10 \mathrm{~V}$ & $\begin{array}{l}\text { Vpp } \\
\text { Offset }\end{array}$ & $\begin{array}{c}110 \mathrm{mV} \\
16 \mathrm{mV}\end{array}$ & $\begin{array}{l}142 \mathrm{mV} \\
27 \mathrm{mV}\end{array}$ & $\begin{array}{c}4.16 \\
-642 \mathrm{mV}\end{array}$ & $\begin{array}{c}4.88 \\
-805 \mathrm{mV}\end{array}$ & $\begin{array}{c}1.76 \\
930 \mathrm{mV}\end{array}$ \\
\hline+-15 & $\begin{array}{l}\text { Vpp } \\
\text { Offset }\end{array}$ & $\begin{array}{c}138 \mathrm{mV} \\
16 \mathrm{mV}\end{array}$ & $\begin{array}{l}144 \mathrm{mV} \\
26 \mathrm{mV}\end{array}$ & $\begin{array}{c}4.16 \\
-634 m V\end{array}$ & $\begin{array}{c}4.88 \\
-800 \mathrm{mV}\end{array}$ & $\begin{array}{c}1.76 \\
926 \mathrm{mV}\end{array}$ \\
\hline
\end{tabular}

\section{Test 2}

Se realiza la prueba esta vez, con una señal de testeo de: $356 \mathrm{~Hz}, 6.5 \mathrm{mV}$, Senoidal, offset de entrada: $5 \mathrm{mV}$

Tabla 2. Medición de la Salida test 2 por cada etapa

\begin{tabular}{|c|c|c|c|c|c|c|}
\hline $\begin{array}{c}\text { Voltaje de } \\
\text { alimentación }\end{array}$ & & $\begin{array}{c}\text { 1 ra } \\
\text { etapa }\end{array}$ & $\begin{array}{c}\text { 2da } \\
\text { etapa }\end{array}$ & $\begin{array}{c}\text { 3ra } \\
\text { etapa }\end{array}$ & 4ta etapa & $\begin{array}{c}\text { Después del } \\
\text { offset }\end{array}$ \\
\hline$+-3.3 \mathrm{~V}$ & $\begin{array}{c}\mathrm{Vpp} \\
\text { Offset }\end{array}$ & $\begin{array}{c}96 \mathrm{mV} \\
22 \mathrm{mV}\end{array}$ & $\begin{array}{c}130 \mathrm{mV} \\
32 \mathrm{mV}\end{array}$ & $\begin{array}{c}4.16 \\
-660 \mathrm{mV}\end{array}$ & $\begin{array}{c}4.88 \\
-825 \mathrm{mV}\end{array}$ & $\begin{array}{c}1.76 \mathrm{~V} \\
916 \mathrm{mV}\end{array}$ \\
\hline$+-10 \mathrm{~V}$ & $\mathrm{Vpp}$ & $92 \mathrm{mV}$ & $132 \mathrm{mV}$ & $\begin{array}{c}4.12 \mathrm{~V} \\
-600 \mathrm{mVp}\end{array}$ & $\begin{array}{c}4.88 \mathrm{~V} \\
-762 \mathrm{mVpp}\end{array}$ & $\begin{array}{c}1.76 \mathrm{~V} \\
950 \mathrm{mV}\end{array}$ \\
\hline
\end{tabular}




\section{Test 3}

Se realiza la prueba esta vez, con una señal de testeo de: $356 \mathrm{~Hz}, 6.5 \mathrm{mV}$,

Senoidal, offset de entrada $-5 \mathrm{mV}$

Tabla 3. Medición de la Salida test 3 por cada etapa

\begin{tabular}{|c|c|c|c|c|c|c|}
\hline $\begin{array}{c}\text { Voltaje de } \\
\text { alimentación }\end{array}$ & & $\begin{array}{c}\text { 1ra } \\
\text { etapa }\end{array}$ & $\begin{array}{l}2 d a \\
\text { etapa }\end{array}$ & $\begin{array}{l}\text { 3ra } \\
\text { etapa }\end{array}$ & $\begin{array}{c}4 \text { ta } \\
\text { etapa }\end{array}$ & $\begin{array}{c}\text { Después del } \\
\text { offset }\end{array}$ \\
\hline$+-3.3 \mathrm{~V}$ & $\begin{array}{c}\text { Vpp } \\
\text { Offset }\end{array}$ & $\begin{array}{l}90 \mathrm{mV} \\
22 \mathrm{mV}\end{array}$ & $\begin{array}{c}130 \mathrm{mV} \\
30 \mathrm{mV}\end{array}$ & $\begin{array}{c}4.16 \mathrm{~V} \\
-660 \mathrm{mV}\end{array}$ & $\begin{array}{c}4.88 \mathrm{~V} \\
-822 \mathrm{mV}\end{array}$ & $\begin{array}{c}1.64 \mathrm{~V} \\
920 \mathrm{mV}\end{array}$ \\
\hline$+-10 \mathrm{~V}$ & $\begin{array}{c}\text { Vpp } \\
\text { Offset }\end{array}$ & $\begin{array}{l}92 \mathrm{mV} \\
18 \mathrm{mV}\end{array}$ & $\begin{array}{c}130 \mathrm{mV} \\
24 \mathrm{mV}\end{array}$ & $\begin{array}{c}4.16 \mathrm{~V} \\
-480 \mathrm{mV}\end{array}$ & $\begin{array}{l}4.88 \mathrm{~V} \\
-650 \mathrm{mV}\end{array}$ & $\begin{array}{l}1.78 \mathrm{~V} \\
1.02 \mathrm{~V}\end{array}$ \\
\hline+-15 & $\begin{array}{c}\text { Vpp } \\
\text { Offset }\end{array}$ & $\begin{array}{l}96 \mathrm{mV} \\
18 \mathrm{mV}\end{array}$ & $\begin{array}{c}128 \mathrm{mV} \\
24 \mathrm{mV}\end{array}$ & $\begin{array}{c}4.16 \\
-510 m V\end{array}$ & $\begin{array}{c}4.88 \\
-683 m V\end{array}$ & $\begin{array}{c}1.76 \\
970 \mathrm{mV}\end{array}$ \\
\hline
\end{tabular}

\section{Test 4}

Se realiza la prueba esta vez, con una señal de testeo de: $356 \mathrm{~Hz}, 6.5 \mathrm{mV}$,

Senoidal, offset de entrada $10 \mathrm{mV}$

Tabla 4. Medición de la Salida test 4 por cada etapa

\begin{tabular}{|c|c|c|c|c|c|c|}
\hline $\begin{array}{c}\text { Voltaje de } \\
\text { alimentación }\end{array}$ & & $\begin{array}{c}\text { 1ra } \\
\text { etapa }\end{array}$ & $\begin{array}{c}\text { 2da } \\
\text { etapa }\end{array}$ & $\begin{array}{c}\text { 3ra } \\
\text { etapa }\end{array}$ & $\begin{array}{c}\text { 4ta } \\
\text { etapa }\end{array}$ & $\begin{array}{c}\text { Después del } \\
\text { offset }\end{array}$ \\
\hline$+-3.3 \mathrm{~V}$ & $\begin{array}{c}\mathrm{Vpp} \\
\text { Offset }\end{array}$ & $\begin{array}{c}92 \mathrm{mV} \\
+18 \mathrm{mV}\end{array}$ & $\begin{array}{c}130 \mathrm{mV} \\
+24 \mathrm{mV}\end{array}$ & $\begin{array}{c}4.16 \\
-580 \mathrm{mV}\end{array}$ & $\begin{array}{c}4.88 \mathrm{~V} \\
-750 \mathrm{mV}\end{array}$ & $\begin{array}{c}1.64 \mathrm{~V} \\
990 \mathrm{mV}\end{array}$ \\
\hline
\end{tabular}




\section{Test 5}

Se realiza la prueba esta vez, con una señal de testeo de: $356 \mathrm{~Hz}, 6.5 \mathrm{mV}$,

Senoidal, offset de entrada $-10 \mathrm{mV}$

Tabla 5. Medición de la Salida test 5 por cada etapa

\begin{tabular}{|c|c|c|c|c|c|c|}
\hline $\begin{array}{c}\text { Voltaje de } \\
\text { alimentación }\end{array}$ & & $\begin{array}{c}\text { 1ra } \\
\text { etapa }\end{array}$ & $\begin{array}{c}\text { 2da } \\
\text { etapa }\end{array}$ & $\begin{array}{c}\text { 3ra } \\
\text { etapa }\end{array}$ & $\begin{array}{c}\text { 4ta } \\
\text { etapa }\end{array}$ & $\begin{array}{c}\text { Después del } \\
\text { offset }\end{array}$ \\
\hline$+-3.3 \mathrm{~V}$ & $\begin{array}{c}\mathrm{Vpp} \\
\text { Offset }\end{array}$ & $\begin{array}{c}96 \mathrm{mV} \\
+18 \mathrm{mV}\end{array}$ & $\begin{array}{c}130 \mathrm{mV} \\
+26 \mathrm{mV}\end{array}$ & $\begin{array}{c}4.16 \\
-560 \mathrm{mV}\end{array}$ & $\begin{array}{c}4.88 \mathrm{~V} \\
-730 \mathrm{mV}\end{array}$ & $\begin{array}{c}1.68 \mathrm{~V} \\
990 \mathrm{mV}\end{array}$ \\
\hline
\end{tabular}

De cada uno de los tests se extraen gráficos para una rápida visualización del comportamiento del offset en el circuito de acondicionamiento.

Offset vs etapa, para valores de offset de entrada de $-10 \mathrm{mV},-5 \mathrm{mV},+-1 \mathrm{mV},+5 \mathrm{mV}$, $+10 \mathrm{mV}$

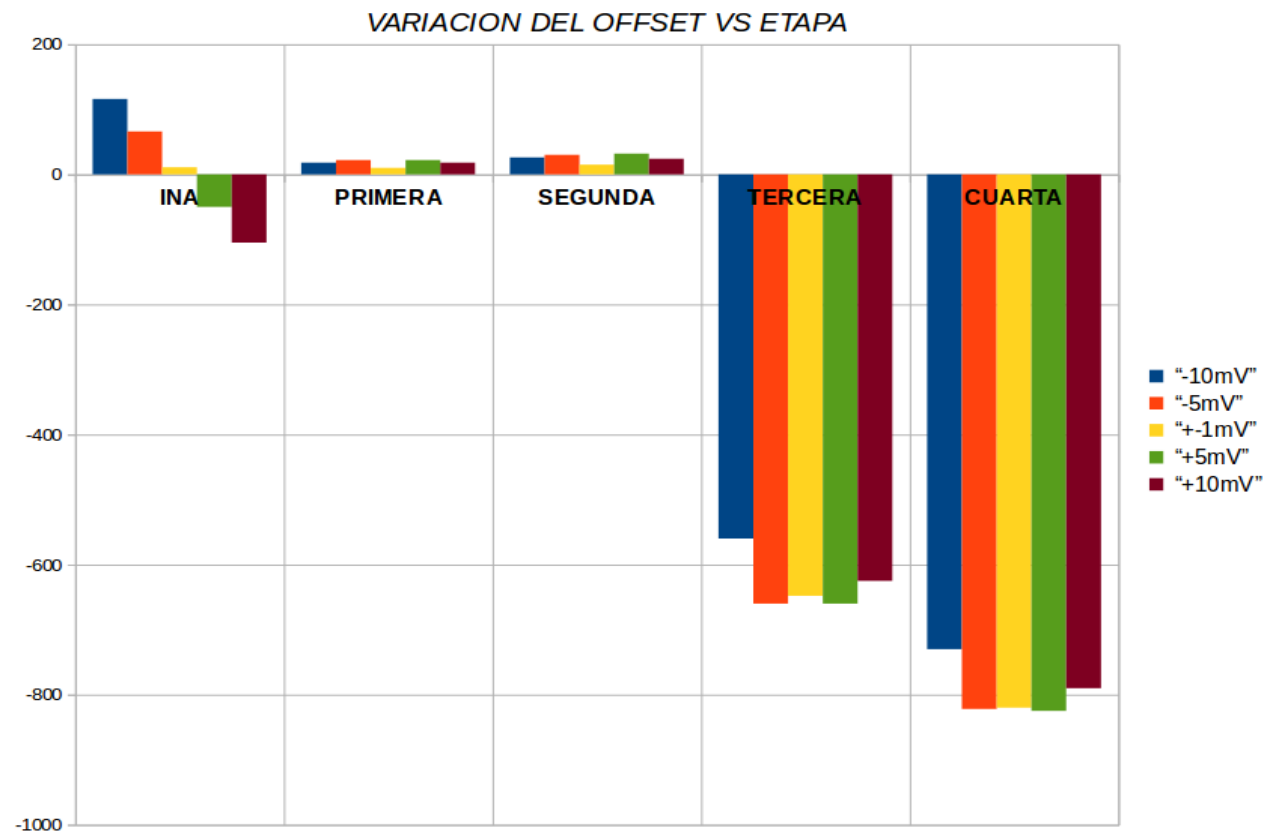

Figura 3. Offset versus etapa, para valores de offset de entrada de $-10 \mathrm{mV},-5 \mathrm{mV}$, $+-1 m V,+5 m V,+10 m V$ 
Tabla 6. Medición por cada etapa de amplificación utilizando señales de entrada de diferente frecuencia.

\begin{tabular}{|l|c|c|c|c|c|c|}
\hline Voltaje de alimentación & & $\begin{array}{c}\text { 1 ra } \\
\text { etapa }\end{array}$ & $\begin{array}{c}\text { 2da } \\
\text { etapa }\end{array}$ & $\begin{array}{c}\text { 3ra } \\
\text { etapa }\end{array}$ & $\begin{array}{c}\text { 4ta } \\
\text { etapa }\end{array}$ & $\begin{array}{c}\text { Después } \\
\text { del offset }\end{array}$ \\
\hline $\begin{array}{l}\text { Señal: } 40 \mathrm{~Hz}, \text { Offset: } \\
\text { 60uV, Vpp: } 6.35 \mathrm{mV}\end{array}$ & $\begin{array}{c}\text { Vpp } \\
\text { Offset }\end{array}$ & $\begin{array}{c}88 \mathrm{mV} \\
8.5 \mathrm{mV}\end{array}$ & $\begin{array}{c}124 \mathrm{mV} \\
16 \mathrm{mV}\end{array}$ & $\begin{array}{c}4.04 \mathrm{~V} \\
-448 \mathrm{mV}\end{array}$ & $\begin{array}{c}5.20 \mathrm{~V} \\
-611 \mathrm{mV}\end{array}$ & $\begin{array}{c}1.76 \mathrm{~V} \\
970 \mathrm{mV}\end{array}$ \\
\hline $\begin{array}{l}\text { Señal: } 20 \mathrm{~Hz}, \\
\text { Offset:+-20uV, Vpp: } \\
\text { 6.35mV }\end{array}$ & $\begin{array}{c}\text { Vpp } \\
\text { Offset }\end{array}$ & $\begin{array}{c}52 \mathrm{mV} \\
17 \mathrm{mV}\end{array}$ & $\begin{array}{c}80 \mathrm{mV} \\
25 \mathrm{mV}\end{array}$ & $\begin{array}{c}2.76 \mathrm{~V} \\
-460 \mathrm{mV}\end{array}$ & $\begin{array}{c}3.60 \mathrm{~V} \\
-625 \mathrm{mV}\end{array}$ & $\begin{array}{c}1.24 \mathrm{~V} \\
968 \mathrm{mV}\end{array}$ \\
\hline $\begin{array}{l}\text { Señal: } 5 \mathrm{~Hz}, \\
\text { Offset:+-1mV }\end{array}$ & $\begin{array}{l}\text { Vpp } \\
\text { Vpp: } 6.35 \mathrm{mV},\end{array}$ & $\begin{array}{c}7 \mathrm{mVpp} \\
17 \mathrm{mV}\end{array}$ & $\begin{array}{c}8 \mathrm{mV} \\
24 \mathrm{mV}\end{array}$ & $\begin{array}{c}320 \mathrm{mV} \\
-500 \mathrm{mV}\end{array}$ & $\begin{array}{c}416 \mathrm{mV} \\
-660 \mathrm{mV}\end{array}$ & $\begin{array}{c}118 \mathrm{mV} \\
950 \mathrm{mV}\end{array}$ \\
\hline $\begin{array}{l}\text { Señal: } 0.1 \mathrm{~Hz}, \\
\text { Offset:+-1mV,Vpp: }\end{array}$ & Offs & $2.5 \mathrm{mV}$ & $2 \mathrm{mV}$ & $0+-20 \mathrm{mV}$ & $0+20 \mathrm{mV}$ & $0+20 \mathrm{mV}$ \\
$6.35 \mathrm{mV}$ & $17 \mathrm{mV}$ & $24 \mathrm{mV}$ & $-480 \mathrm{mV}$ & $-650 \mathrm{mV}$ & $950 \mathrm{mV}$ \\
\hline
\end{tabular}

\section{Comportamiento del filtro pasa altas:}

\section{Vpp vs frecuencia}

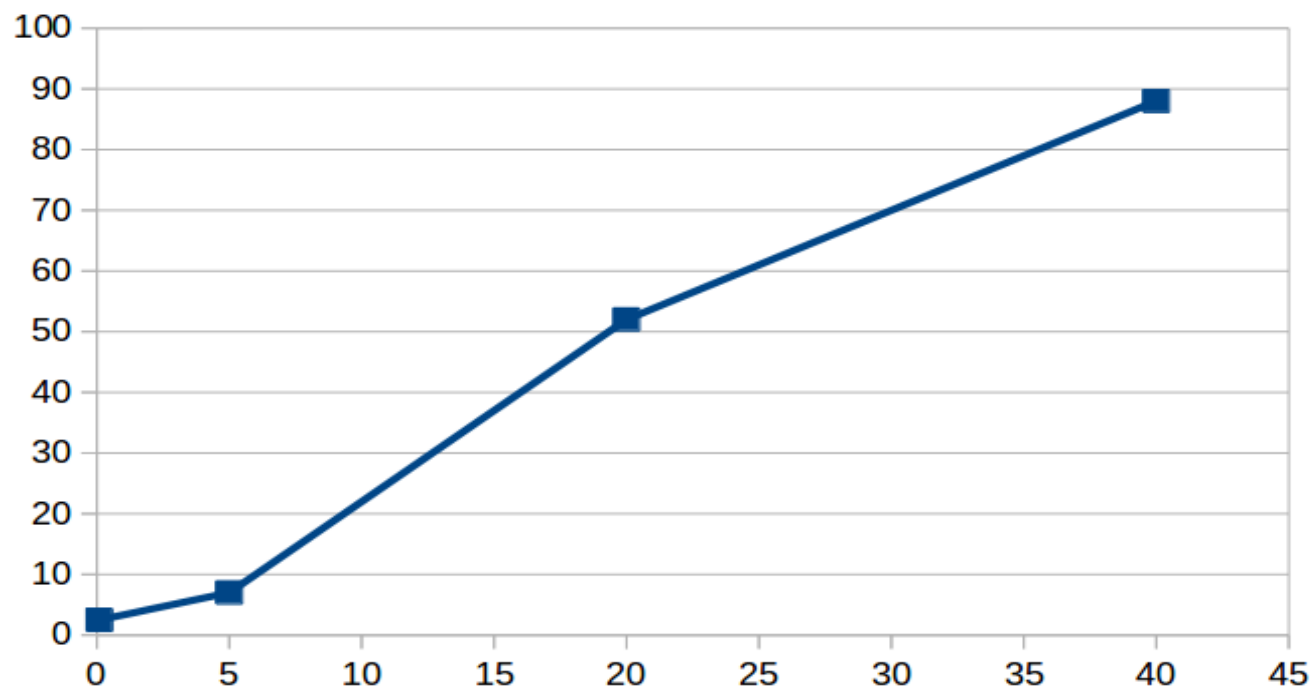

Figura 4. Voltage pico a pico (Vpp) versus frecuencia en la primera etapa 


\section{Comportamiento del filtro pasa altas en baja frecuencia por etapas}

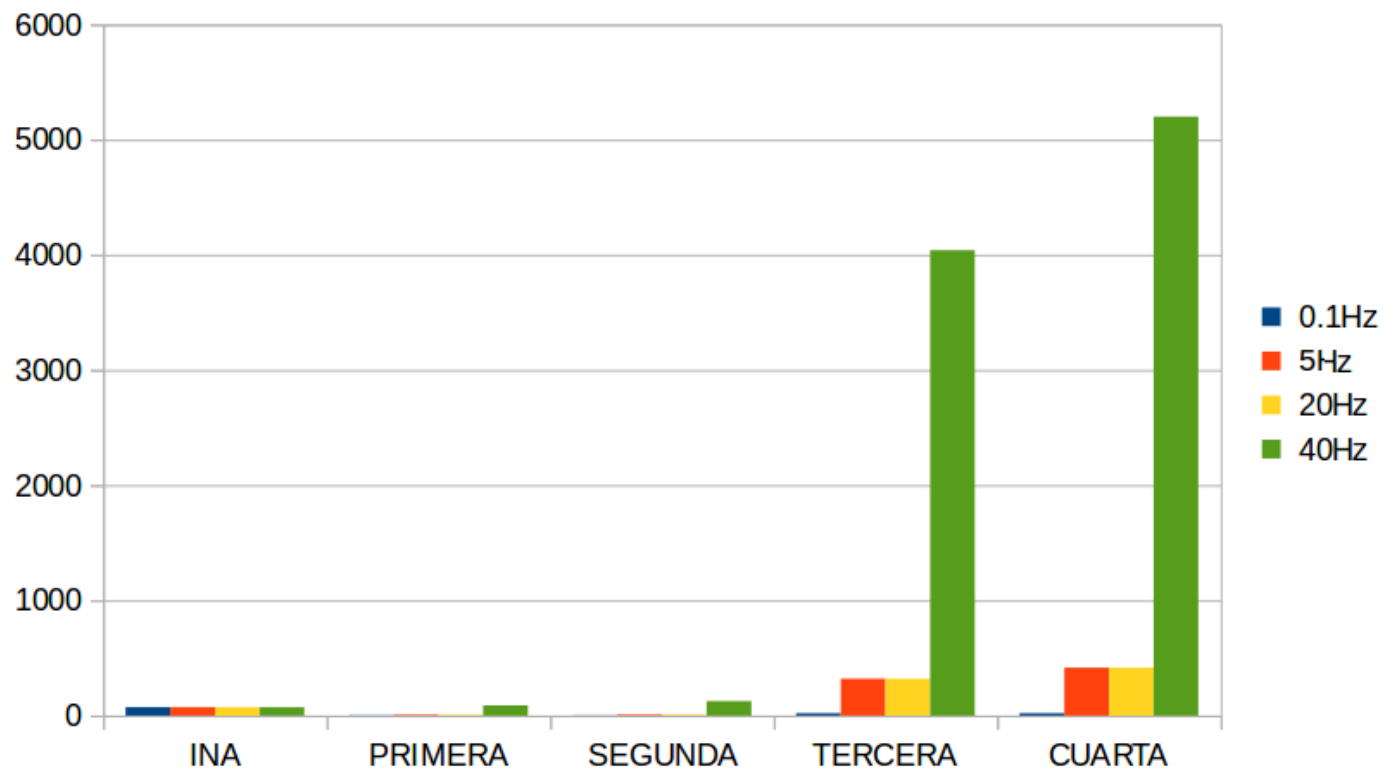

Figura 5. Voltage pico a pico (Vpp) versus frecuencia en cada etapa

De las figuras anteriores, se puede observar que la señal es suprimida en mayor proporción mientras más baja es la frecuencia comparado con frecuencia de corte $(2 \mathrm{~Hz})$.

Nótese que esta atenuación es para el voltaje de pico a pico, el cual da la noción de que el filtro pasa altas está cumpliendo con su trabajo. 
offset vs frecuencia

SALIDA DEL FILTRO PASAALTAS

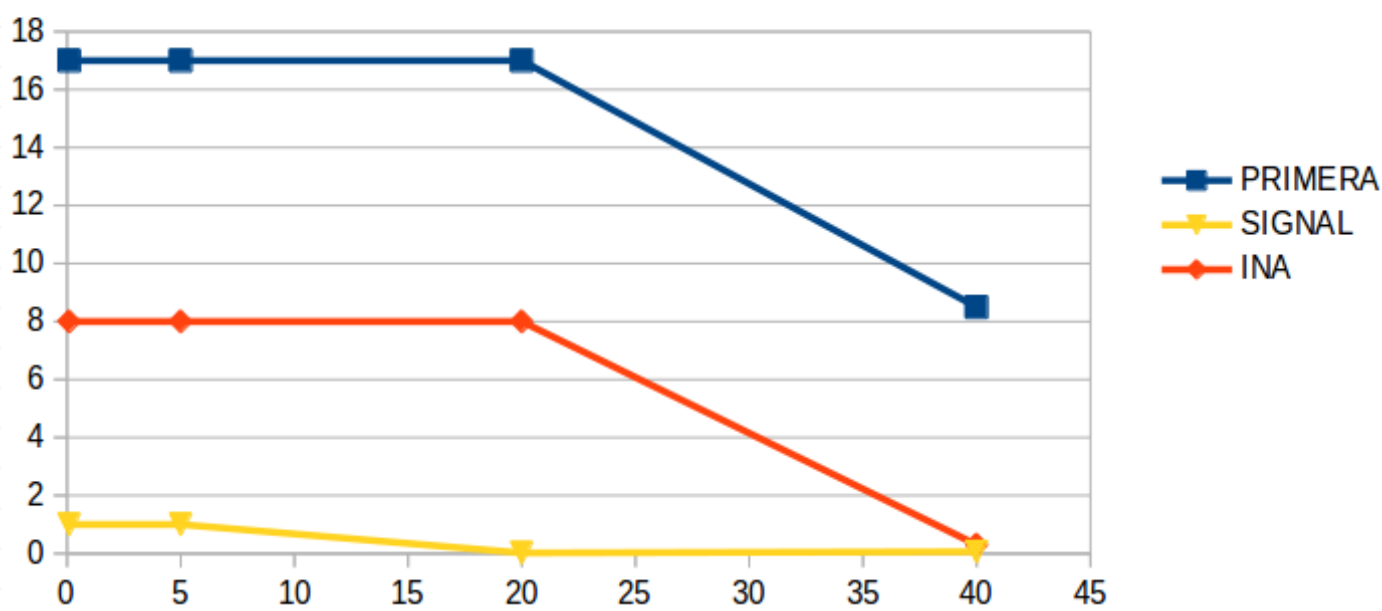

Figura 6. Relación del offset vs frecuencia a la salida del filtro pasa alta

Nótese que para el caso del offset este no es disminuido por el filtro pasa altas (Primera Etapa), aparentemente este offset es incrementado proporcionalmente en todos los puntos.

A continuación, se realiza la toma de datos para baja frecuencia con offset de escala superior para así poder ver el comportamiento de la atenuación de offset en baja frecuencia. 


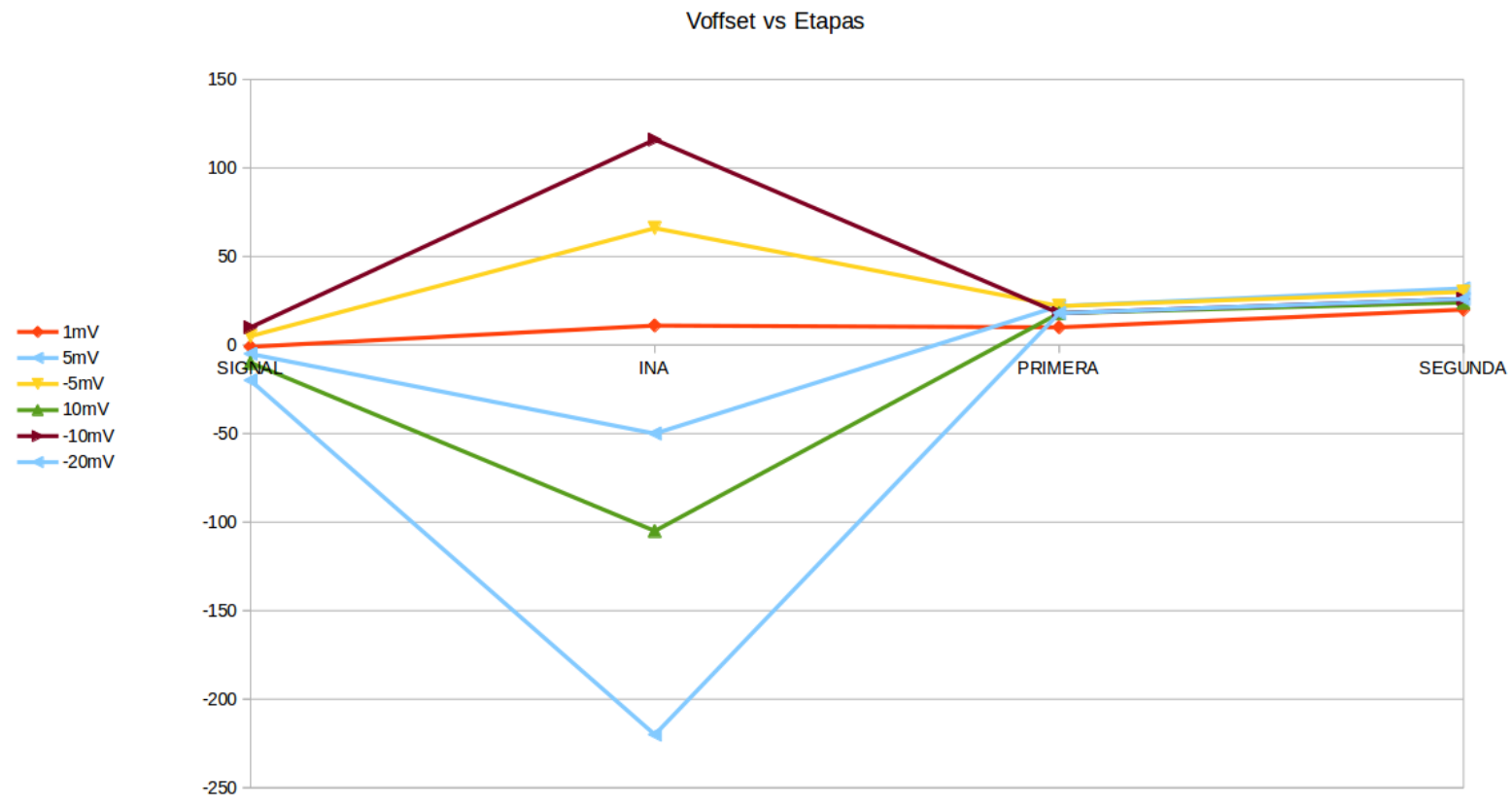

Figura 7. Análisis del offset versus frecuencia en la primera etapa con una señal de entrada $356 \mathrm{~Hz}, 6.5 \mathrm{mVpp}$, senoidal. El offset de entrada varía de acuerdo a la leyenda.

Se puede observar que el offset de la señal es amplificado por el amplificador instrumental en proporción a su ganancia (11), en general estos offset's amplificados son suprimidos en gran parte por el filtro pasa altas, sin embargo este filtro deja un pequeño offset de hasta $24 \mathrm{mV}$ aproximadamente. El cual es amplificado en las siguientes etapas donde este offset se vuelve muy notorio.

En este caso un offset de $18 \mathrm{mV}-24 \mathrm{mV}$ a la salida del EMG podría ser despreciado fácilmente.

Se puede evaluar la utilidad de colocar 2 filtros pasa bajas $700 \mathrm{~Hz}$ y $500 \mathrm{~Hz}$, se podría utilizar solo 1 filtro pasa bajas de $500 \mathrm{~Hz}$ y utilizar 1 filtro pasa alta al inicio y otro al final en caso de ser necesario 1 filtro pasa altas al inicio de la amplificación. 


\section{Evolución del Offset por etapa a distintas frecuencias (bajas)}

La imagen anterior muestra el comportamiento del offset por etapa, para distintas frecuencias. Se puede observar una subida del offset del amplificador instrumental a la primera etapa, lo cual apoyaría la idea de un offset a la salida de la primera etapa independientemente del offset de entrada.

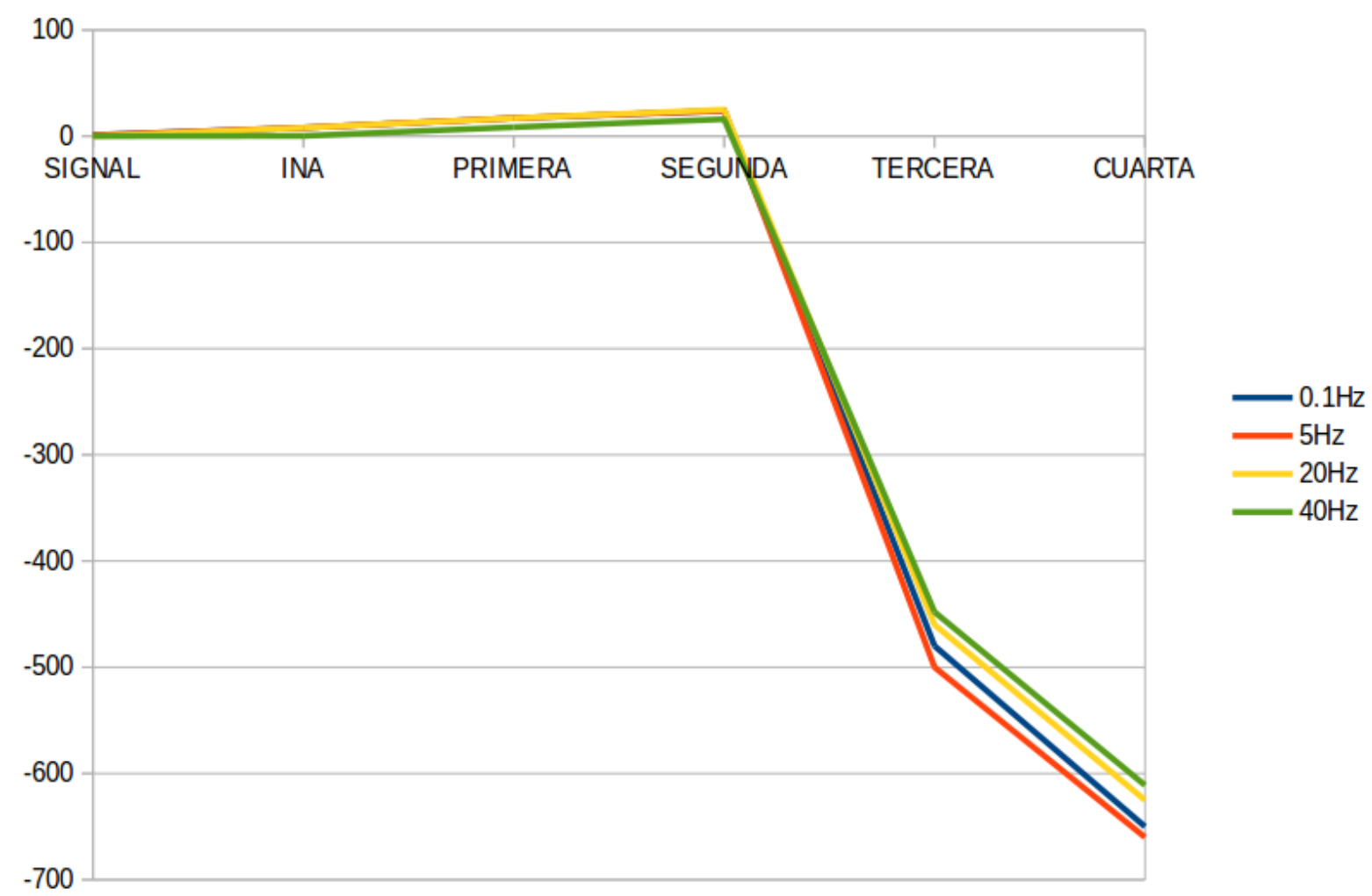

Figura 8. Análisis del offset a diferentes frecuencias de entrada

\section{CONCLUSIONES Y DISCUSIÓN}

El presente estudio presenta resultados preliminares en la evaluación y depuración del circuito de acondicionamiento utilizado en el diseño de un sensor EMG superficial de electrodos secos.El amplificador instrumental amplifica el offset de la señal aproximadamente en: $-G^{*} V$ offset, donde $G$ es el valor de la ganancia en esta etapa. En el circuito analizado, el offset para la segunda y posteriores etapas se está 
amplificando en proporción a sus ganancias, de acuerdo a la salida de la primera etapa.

A partir del estudio realizado se pueden evidenciar algunas conclusiones:

En primer lugar, cada una de las etapas cumple su cometido de acuerdo a las simulaciones realizadas. Segundo, la señal senoidal de entrada para las frecuencias dentro del espectro útil del circuito de acondicionamiento $(20-500 \mathrm{~Hz})$ no se ve alterada según la inspección visual.Tercero, las señales menores a $20 \mathrm{~Hz}$ son atenuadas en la segunda etapa del filtro pasa alta. Sin embargo, como se puede evidenciar en las tablas, en la tercera etapa también ocurre una atenuación. Esto se debe a que la señal contiene ruido en alta frecuencia el cual es depurado en la etapa 3 (filtro pasa baja), razón por la cual se aprecia una caída de voltaje.

El presente estudio toma en cuenta solo un subsistema que compre el funcionamiento de un sensor electromiográfico. Otros aspectos como el material del electrodo pueden complementar el análisis y validación del sensor. En futuros estudios se puede evaluar una mejor selección del material a ser utilizado como electrodo seco, en el mercado existen diversas alternativas y aleaciones de aceros que pueden proveer una mejor relación de conductividad piel-electrodo y así maximizar la fidelidad de la señal adquirida. En este caso, para asegurar la fijación del electrodo a la piel, otros electrodos comerciales utilizan una pegatina en forma de marco alrededor del sensor, la cual es removible, reemplazándose en cada uso. Esta pegatina asegura la fijación del electrodo y sería recomendable fabricar una similar para el sensor diseñado en el presente estudio.

Asimismo, futuros trabajos pueden incluir el encapsulamiento del sistema adicionando una caja de Faraday y manufactura aditiva que sirva para aislar el circuito de posibles interferencias electromagnéticas.

Si bien es cierto, el presente estudio toma en cuenta el análisis de circuitos analógicos, no se descarta que el uso de filtros digitales de altos órdenes pueda 
mejorar la calidad de la señal adquirida; sin embargo, deberá evaluarse el costo computacional y la latencia originada debido a la adición de estas etapas.

El presente trabajo busca contribuir al estado del arte de los sensores electromiográficos desde un punto de vista técnico y de fabricación. La meta final en todo sensor que pueda ser de utilidad y escala masiva es que cumpla con los requerimientos mínimos para su uso en personas que necesiten de un análisis muscular, en especial personas con discapacidad motora.

En particular, una de las discapacidades motoras con mayor índice de personas afectadas es la amputación de uno de los miembros producto de enfermedad o trauma (Pet, 2016). La solución propuesta para los usuarios que sufren algún tipo de amputación, es el reemplazo del miembro por un miembro artificial también conocido como prótesis.

El desarrollo de prótesis en los últimos años se ha enfocado en mejorar la funcionalidad y apariencia del miembro artificial. En la actualidad, existen diferentes alternativas de prótesis para los usuarios. Desde opciones puramente cosméticas, prótesis mecánicas y recientemente prótesis biónicas, controladas por señales musculares o mioeléctricas. Diferentes empresas comercializan este tipo de prótesis (BeBionics, iLimb, Touch Bionics, Vincent) con precios desde los 60 mil dólares (Hofmann, 2017).

El campo del desarrollo de prótesis sigue en constante crecimiento, tanto a nivel de investigación como a nivel comercial. Dentro de los Antecedentes peruanos, el departamento de biomecánica del Instituto Nacional de Rehabilitación desarrolla prótesis mecánicas con un costo de 7 mil soles.

Asimismo, existen iniciativas que prometen abaratar costos de las prótesis a través de manufactura aditiva. Dentro del marco de prótesis biónicas, existen proyectos de investigación orientados al desarrollo de prótesis robóticas en el Perú por grupos de diferentes universidades. 
Sin embargo, los trabajos a futuro de estos proyectos, así como los costos aterrizados hacia una propuesta comercial no se encuentran totalmente definidos hasta el momento de la redacción de este trabajo. Uno de los componentes importantes sigue siendo el sensor muscular a ser utilizado en estas prótesis, acompañado de algoritmos de clasificación que permitan interpretar los movimientos de la persona para que así pueda abrir o cerrar la mano con tan solo pensarlo ( $\mathrm{Xu}$, 2017).

El diseño de algoritmos de clasificación avanzados y adaptativos basados en redes convolucionadas y filtros no líneas utilizando diferentes características de la señal puede mejorar la señal (Altin, 2016) y su estudio está abierto a potenciales estudiantes interesados en el uso de los sensores electromiográficos para el estudio y aplicación de estas en la mejora de la calidad de vida de las personas con discapacidad motora.

\section{REFERENCIAS CONSULTADAS}

1. Altin, C., \& Er, O. (2016). Comparison of different time and frequency domain feature extraction methods on elbow gesture's EMG. European journal of interdisciplinary studies, 2(3), 35-44.

2. Beck, T. W., Housh, T. J., Cramer, J. T., Malek, M. H., Mielke, M., Hendrix, R., \& Weir, J. P. (2007). A comparison of monopolar and bipolar recording techniques for examining the patterns of responses for electromyographic amplitude and mean power frequency versus isometric torque for the vastus lateralis muscle. Journal of neuroscience methods, 166(2), 159-167.

3. Brown, B. H., Smallwood, R. H., Barber, D. C., Lawford, P. V., \& Hose, D. R. (2017). Medical physics and biomedical engineering. CRC Press.

4. Catarino, A. P., Rocha, A., Carvalho, H., \& Ferreira, F. N. (2017). Textile based electrodes for ECG and EMG measurements. In Proceedings of the ITMC2017-International Conference on Intelligent Textiles and Mass Customisation. 
5. Farina, D., Stegeman, D. F., \& Merletti, R. (2016). Biophysics of the Generation of EMG Signals. Surface Electromyography: Physiology, Engineering, and Applications, 1-24.

6. Hofmann, M., Harris, J., Hudson, S. E., \& Mankoff, J. (2016, May). Helping hands: Requirements for a prototyping methodology for upper-limb prosthetics users. In Proceedings of the $2016 \mathrm{CHI}$ Conference on Human Factors in Computing Systems (pp. 1769-1780). ACM.

7. Merletti, R., Botter, A., \& Barone, U. (2016). Detection and conditioning of surface EMG signals. In Surface electromyography: Physiology, engineering, and applications. IEEE Press-John Wiley \& Sons, Inc..

8. Nuyujukian, P., Sanabria, J. A., Saab, J., Pandarinath, C., Jarosiewicz, B., Blabe, C. H., ... \& Hochberg, L. R. (2018). Cortical control of a tablet computer by people with paralysis. PloS one, 13(11), e0204566.

9. Pet, M. A., Morrison, S. D., Mack, J. S., Sears, E. D., Wright, T., Lussiez, A. D., ... \& Kung, T. A. (2016). Comparison of patient-reported outcomes after traumatic upper extremity amputation: replantation versus prosthetic rehabilitation. Injury, 47(12), 2783-2788.

10. Xu, W., Sun, Y., \& Li, C. (2017, October). Research on EMG Signal Extraction Based on Biomimetic Hand. In 7th International Conference on Management, Education, Information and Control (MEICI 2017). Atlantis Press.

\section{REFERENCES CONSULTED}

1. Altin, C., \& Er, O. (2016). Comparison of different time and frequency domain feature extraction methods on elbow gesture's EMG. European journal of interdisciplinary studies, 2 (3), 35-44.

2. Beck, T. W., Housh, T. J., Cramer, J. T., Malek, M. H., Mielke, M., Hendrix, R., \& Weir, J. P. (2007). A comparison of monopolar and bipolar recording techniques for examining the patterns of responses for electromyographic amplitude and mean power frequency versus isometric torque for the vastus lateralis muscle. Journal of neuroscience methods, 166 (2), 159-167. 
3. Brown, B. H., Smallwood, R. H., Barber, D. C., Lawford, P. V., \& Hose, D. R. (2017). Medical physics and biomedical engineering. CRC Press

4. Catarino, A. P., Rocha, A., Carvalho, H., \& Ferreira, F. N. (2017). Textile based electrodes for ECG and EMG measurements. In Proceedings of the ITMC2017-International Conference on Intelligent Textiles and Mass Customization.

5. Farina, D., Stegeman, D. F., \& Merletti, R. (2016). Biophysics of the Generation of EMG Signals. Surface Electromyography: Physiology, Engineering, and Applications, 1-24.

6. Hofmann, M., Harris, J., Hudson, S. E., \& Mankoff, J. (2016, May). Helping hands: Requirements for a prototyping methodology for upper-limb prosthetics users. In Proceedings of the $2016 \mathrm{CHI}$ Conference on Human Factors in Computing Systems (pp. 1769-1780). ACM

7. Merletti, R., Botter, A., \& Barone, U. (2016). Detection and conditioning of surface EMG signals. In Surface electromyography: Physiology, engineering, and applications. IEEE Press-John Wiley \& Sons, Inc ..

8. Nuyujukian, P., Sanabria, J. A., Saab, J., Pandarinath, C., Jarosiewicz, B., Blabe, C. H., ... \& Hochberg, L. R. (2018). Cortical control of a tablet computer by people with paralysis. PloS one, 13 (11), e0204566.

9. Pet, M. A., Morrison, S. D., Mack, J. S., Sears, E. D., Wright, T., Lussiez, A. D., ... \& Kung, T. A. (2016). Comparison of patient-reported outcomes after traumatic upper extremity amputation: replantation versus prosthetic rehabilitation. Injury, 47 (12), 2783-2788.

10. Xu, W., Sun, Y., \& Li, C. (2017, October). Research on EMG Signal Extraction Based on Biomimetic Hand. In 7th International Conference on Management, Education, Information and Control (MEICI 2017). Atlantis Press 